\title{
Combining multicriteria decision aid and system dynamics for the control of socio-economic processes. An iterative real-time procedure
}

\author{
J.P. Brans ${ }^{\text {a,* }}$, C. Macharis ${ }^{\text {a }}$, P.L. Kunsch ${ }^{b}$, A. Chevalier ${ }^{c}$, M. Schwaninger ${ }^{\text {d }}$

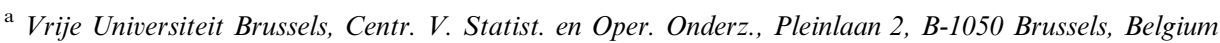 \\ ${ }^{\mathrm{b}}$ Université Libre de Bruxelles, SMG Bd. du Triomphe P.O. Box 210/01, B1050 Brussels, Belgium \\ ${ }^{\mathrm{c}}$ ESCP, Av. de la République 79, 75543 Cédex 11, Paris, France \\ ${ }^{\mathrm{d}}$ Universität St. Gallen, CH-9000, St. Gallen, Switzerland
}

Received 1 March 1997

\begin{abstract}
The paper presents the elements of a new methodology to control complex and hypercomplex socio-economic structures. The control process is iterative, combining the principles of System Dynamics, Control theory and the PROMETHEE Multicriteria Decision Aid (MCDA) methodology. It consists of three main stages: setting up and calibration of a quantitative model, definition of long-term strategies and short-term control. The purpose is to define within a panel of decision makers appropriate strategies towards long-term goals, and to implement suitable control measures. These should in particular help cope with progressive and catastrophic variations in the behaviour of the system. (c) 1998 Published by Elsevier Science B.V. All rights reserved.
\end{abstract}

Keywords: Hypercomplex socio-economic systems; System Dynamics; MCDA PROMETHEE methodology; Long-term planning and real time control

\section{Introduction}

The modelling of a socio-economic system, for the sake of understanding and controlling it, undoubtfully belongs to the class of complex and even hypercomplex problems.

In the present paper, a problem will be called complex if its quantitative modelling requests an

\footnotetext{
${ }^{*}$ Corresponding author. Fax: 322629 2282; e-mail: jpbrans@isl.vub.ac.be.
}

infinite number of interrelated equations to adequately represent the real world situation. The problem is hypercomplex when each differential neighbourhood is already a complex system, all the neighbourhoods being in addition partially independent. ${ }^{1}$

\footnotetext{
${ }^{1}$ There are many other useful definitions of complexity and hypercomplexity, but we think this simple and straightforward definition is able to capture all the other features of a complex system (cf. Lemoigne, 1995).
} 
In such circumstances it seems hopeless to try to assist decision makers with quantitative models. However history has shown in many circumstances that the management of socio-economic systems by mere intuition and qualitative judgment has been insufficient (Forrester, 1968). Forecasts for future evolution must be made available for assisting policy makers. Although this is a hard task due to the hypercomplexity of the systems, it is necessary to provide the decision makers with assessments regarding the future.

This could be illustrated in the critical situation faced by the macro-economic system of the seventies. At that time the necessary political decisions were not taken to avoid the exponential increase of unemployment, public debt and social security expenses. The control methodology, based on System Dynamics and Multicriteria Decision Making, we propose in this paper, could have provided the adequate tool for the analysis of the very first stage of the crisis. It could have been shown that unemployment, public debt and social security expenses were embedded in an exponential increasing positive feedback loop. It would have been necessary to detect the appropriate strategy to compensate this positive growth by adequate negative feedback loops in order to keep the system within reasonable limits. The whole European cultural and democratic process could then possibly have avoided its present major crisis.

In the past years, and even up to now, econometric models and regression analyses have often been used for such purposes. We believe that this approach is not satisfactory. Of course econometric models can accumulate the appropriate historical information providing a precise description of the past. The analysis of the collected data may provide the correct range of values of the important parameters and the correct interactions between variables during the observed period. But as soon as such models are used for predictions, they fail because of the rapid changes in the socio-economic structures. These changes make the predictions useless and decision-making hazardous (Meadows, 1980). No control procedure can be applied.

We strongly believe that a harmonious combination of System Dynamics (see a review by Legas- to et al., 1980) and the Multicriteria Decision Aid (MCDA) PROMETHEE procedure (Brans and Mareschal, 1994) supported by appropriate modelling and simulation can contribute significantly to a proper control of socio-economic systems. It should act in real-time on the very dynamical structure system. Let us call this approach control of structure (Kunsch, 1996).

The purpose is to help decision makers to express their mental representation of the system under study, to structure it, and to integrate it into a causal-loop diagram (Forrester, 1968, 1992). The influence diagram is essential to understanding the relationships between the different elements of the system, in order to design and control for a desirable future. The formalized SD model will be derived from this representation. Once the model has been set up, several strategies can be simulated. These strategies have to be evaluated on several criteria. The PROMETHEE methodology is used to select an appropriate strategy to govern the system. In addition, the modelling approach must evolve in an iterative and interactive fashion, as requested by the basic idea of the control of structure. It must permit to enhance at regular time intervals the vision of the future. This is made possible by the continuous observation of the system. At each reassessment, the mental representation, the influence diagram, and the quantitative model can be modified. The control procedure associated with each reassessment period is called an iteration. Each iteration consists of 11 basic steps enumerated below and described in detail in Section 2.

1. Elaboration of a mental representation.

2. Definition of the system variables, relationships between them and the decision variables to be considered for control; setting up the influence diagram.

3. Identification of the positive and negative feedback loops within the influence diagram.

4. Positioning of watchdogs and control.

5. System dynamics modelling and validation.

6. Generating strategies.

7. Simulation of scenarios, strategic options and long-term test.

8. Ranking and selection using the MCDA PROMETHEE procedure. 
9. Realization of the selected strategy.

10. Definition of a socio-economic core and shortterm control.

11. Analysis of the deviations between plan and actual evolution.

- Next iteration. Branching back.

\section{The methodology: The basic steps of each iteration}

The dynamic control of structure we propose to govern the evolution of socio-economic systems is based on the elaboration of a mental representation of the real world, its translation into an influence diagram, the definition of strategic options, the simulation of these strategies with System Dynamics (SD) and the selection of an appropriate control policy by using the PROMETHEE multicriteria procedure.

The control procedure consists of a succession of iteration periods. Each of them includes the steps already enumerated, and described in more detail here.

\subsection{Step 1: Elaboration of a mental representation}

This is the very important step in the conceptualization of SD modelling as stressed by many authors (see e.g. Randers, 1980; Forrester, 1992). ${ }^{2}$ The purpose is to obtain in a qualitative way a good view of the components of the system and the dynamic interactions. Also external influences have to be considered. Continuous reference should be made to the intuitive thinking and to the mental data base accumulated from past experience and observation. Each decision maker has his own specific mental model, according to his perception of the relevant structures. It is the pur-

\footnotetext{
${ }^{2}$ Several knowledge elicitation methods have been proposed in the SD-literature (see for instance Richardson and Andersen, 1995). Methods from soft OR (Vennix et al., 1990; Eden, 1994) and the Soft System Methodology (Checkland, 1981) were also combined with the System Dynamics approach (see for instance Daellenbach, 1994). In the present paper we are however not going into detail into the conceptualization phase.
}

pose of this first step to aggregate these preferences into an overall mental representation.

Whichever representation is chosen, it will provide to the decision makers a better understanding of the problem. It should include an analysis of the structures of the system in the past (the basic mechanisms) and an educated guess about the expected behavior of the major variables (the reference mode). Together this constitutes the dynamic hypothesis of the study (Randers, 1980). In addition, the decision makers should formulate their projects, which goals they want to attain and consequently which criteria they will use in evaluating the several possible strategies.

\subsection{Step 2: Definition of the system and decision variables. Influence diagram}

The mental representation should name all variables and parameters in order to be able to draw the causal-loop diagram, we name "influence diagram". We briefly recall some definitions which are essentially based on the System Dynamics Methodology (Forrester, 1968).

System variables allow to describe the evolution of the system. Among them state variables have the nature of stocks (e.g. the public debt of a country). These variables accumulate variations in time induced by flow variables (e.g. the yearly expenditures and budget of that country). State variables can be said to represent the memory of the system up to the present time. Some system variables are neither stocks nor flows. They are called auxiliary variables and are connected with other system variables to express combinations or functional relationships.

Decision variables are the policy instruments through which the decision makers can "exogeneously" influence the system. They therefore represent a part of the degrees of freedom in controlling the system. They often have the nature of flows and auxialiary variables. In a simple microeconomic system, decision variables could be investment decision, changes in human resources, yearly marketing expenses, $\mathrm{R}$ and $\mathrm{D}$ expenses, etc.

As a general rule, the set variables as defined in the course of the first iteration will be simplified, 
leaving out the less essential ones. This will permit to avoid too intricate influence diagrams in which essential mechanisms are impossible to grasp. The GAMMA software is an excellent tool for simplifying the diagram (Schwaninger, 1997).

However after each iteration, finer tuning of the model to reality will increase the complexity (see step 11). The number of variables, parameters, connecting arrows and loops will in general increase as time unfolds. The learning feedbackloop due to the modelisation will ease the understanding of the decision makers facing the increased complexity of the model (Sterman, 1994).

\subsection{Step 3: Identification of positive and negative feedback loops}

In order to control the future evolution of the system, it is crucial to detect first the existence of cycles in the influence diagram. Cycles are responsible for feedbacks, in which an action or an influence exerted by one of the system components will finally affect the latter in return, either by amplification or by damping of this original action/influence. (+) Feedback loops imply an amplification of perturbations (increase or decrease) and herewith a divergence from attractors. They are vicious circles or destabilizing loops. (-) Feedback loops on the contrary imply attenuation of perturbations and herewith convergence to a stable attractor. They are goal-seeking loops or stabilizing loops, and they are the very instrument of control.

The main worry of socio-economic decision makers should be to establish the sustainable balance of both types of feedback loops. Indeed, although uncontrolled (+) loops in principle seem to indicate unlimited departure from stability, they will hit some limits in the course of the dynamic evolution.

The purpose of detecting and analyzing feedback loops is clearly to identify on one hand the needs for control, and on the other hand to ascertain the possibility of control mechanisms, in order to prevent all too risky and destructive dynamics. Most important, new connections between variables will have to be pondered, and corrective loops designed. Establishing well-balanced cycles is a must whenever detrimental loops are the alternative, prone to emerge by themselves through "self-organization".

\subsection{Step 4: Positioning of watchdogs and control}

As described above, (+) feedback loops must be properly monitored and brought to an adequate balance by the countervailing action, via intrinsic or extrinsic control loops, i.e. (-) feedback loops.

The monitoring of (+) loops has to be made by defining watchdogs, i.e. important variables which should signal the possible triggering of unfavourable "snowballing" effects when passing some critical thresholds. As an example, a critical liquidity level in the firm would be self-reinforcing due to $(+)$ loops between decreasing liquidity and declining sales figures on one hand, and reduced marketing actions or $\mathrm{R}$ and $\mathrm{D}$ investment on the other hand. Watchdog variables or parameters will be chosen among system variables representing critical assumptions (those assumptions, the non-materialization of which is likely to threaten a strategy) or belonging to critical (+) feedback loops. If necessary, additional auxiliary variables can be inserted to fulfil this function. Sensitivity analysis can be used to detect the thresholds.

The "flickering" of any watchdog in the real system signals potential risk. It provides some information on the importance of this risk and on the priorities for undertaking control actions. Two cases are being considered for these control tasks, depending on the existence or not of a sufficient intrinsic control in the system during a given iteration:

1. Existence of intrinsic control: in this case, intrinsic control loops are supposed to be available in the system. Simulations should show if and under which conditions the equilibrating action of the loops, thanks to appropriate values of some decision variables, would be sufficient to dampen risky evolutions. In addition, extrinsic control would be necessary as described under 2;

2. Extrinsic control has to be built in: absent or insufficient control calls for introducing additional $(-)$ control loops into the system and its model. In this way snowballing $(+)$ feedback loops are 
brought back into balance, and critical variables are kept within reasonable bounds. In practice the loops are equivalent to using adapted socioeconomic instruments, represented in the model by new decision variables. For example a new department for internal communication with all its influences can be introduced into the model and the system.

Quite generally, the design of the control loop will evolve over time from iteration to iteration, as new information about the system is made available by observation, watchdogs are giving warning alarms, or exogeneous events impact the system. During the iteration periods, the structures of the system/model can be adapted and control loops be redesigned according to the modified knowledge and state of affairs. In many cases both types of control will be conjointly applicable.

\subsection{Step 5: System dynamics modelling and validation}

By means of the qualitative representation of the influence diagram, the socio-economic system can be visualized as an organized assembly of interrelated parameters and variables. Unfortunately this entangled loop structure makes a reliable judgement about the dynamic behaviour of the system at hand, on a merely intuitive basis unlikely (Forrester, 1987). This "unpredictability" is reinforced by the nonlinear character of many interactions within the diagram. The translation of the influence diagram into a simulation model using the System Dynamics approach is necessary to simulate the future.

Model building is supported by software packages such as STELLA/ithink (High Performance, 1994). The system of multiple differential equations can be run from given initial conditions to a defined long-term horizon $(T)$, in order to simulate future behaviour.

The validation of models is very important because model quality is essential to the conclusions on subject matters, strategies and their successful realization. Validating SD models is a sophisticated issue in itself. The use of historical and statistical data is part of the process, but validation, in this case, includes a whole set of tests (see in particular Forrester and Senge, 1980; Barlas, 1996), namely

1. direct structure tests,

2. structure-oriented behaviour tests,

3. behaviour pattern tests.

Much of the validation procedures can be considered in step 5, but they should not be confined to it. In particular, the theoretical structure test should already start throughout steps 1-4.

\subsection{Step 6: Generating strategies}

In this step, the decision makers develop strategies to be possibly adopted. They may use the support of external consultants for some specific specialized or technical aspects.

Prior to the strategy, different scenarios of "virtual realities" can be explored, of which the probable and desirable ones will be chosen for further pursuit. Then, a set of strategies apt to bring these scenarios about has to be designed. Generating scenarios and strategies goes "hand in hand", interactively, with pertinent simulations (step 7). While working out scenarios and strategies, appropriate space should be given as much to intuition, foreseeing capabilities and creativity as to the mental representation and the quantitative model (Forrester, 1992; Randers, 1980). Brainstorming meetings, supported by Group Decision Support technologies can enrich the procedure we propose (Vennix, 1996; Kenis, 1995).

Assume $N$ possible strategies, are proposed during the group session.

$S_{1}, \ldots, S_{i}, \ldots, S_{N} \quad i=1,2, \ldots, N$.

As argued before (step 4) these strategies can be a simple setting of the decision variables or a more fundamental change of the structure of the model.

Advanced modelling tools, e.g. the VENSIM software, open space for parameter simulation, which actually involves a calculus of high numbers of variants (or "mutants"), without overloading decision makers with excessive complexity. Moreover, the PROMCALC software (see step 8) enables the user to cope with several strategies at one time. 
2.7. Step 7: Simulation of scenarios, strategic options, and long-term test

As mentioned, step 7 is executed in iterations with step 6 . The differential equations set up in step 5 are integrated for each scenario or strategy over a long-term horizon $(0, T)$. $T$ should be chosen large enough to be able to assess the long-term behaviours. The user-friendly System Dynamics computer packages, already named under step 5, are available for this purpose.

For each strategy the results of the simulations are made available in the form of the long-term time paths of the system variables. Some of those variables are interpreted as objectives or goals to be satisfied in the long term, giving to decision makers the basis for comparing the merits of different strategies (see Fig. 1).

As an example of goals in systems, the convergence criterion of $3 \%$ defined by the Council of Ministers of the European Union in Maastricht (the yearly increase of the public debt must not be higher than $3 \%$ of the GNP) has nowadays (mid-1996) been accepted as a necessary condition for European countries to be able to join the European Monetary Union (EMU). It has therefore become an objective of major importance for most

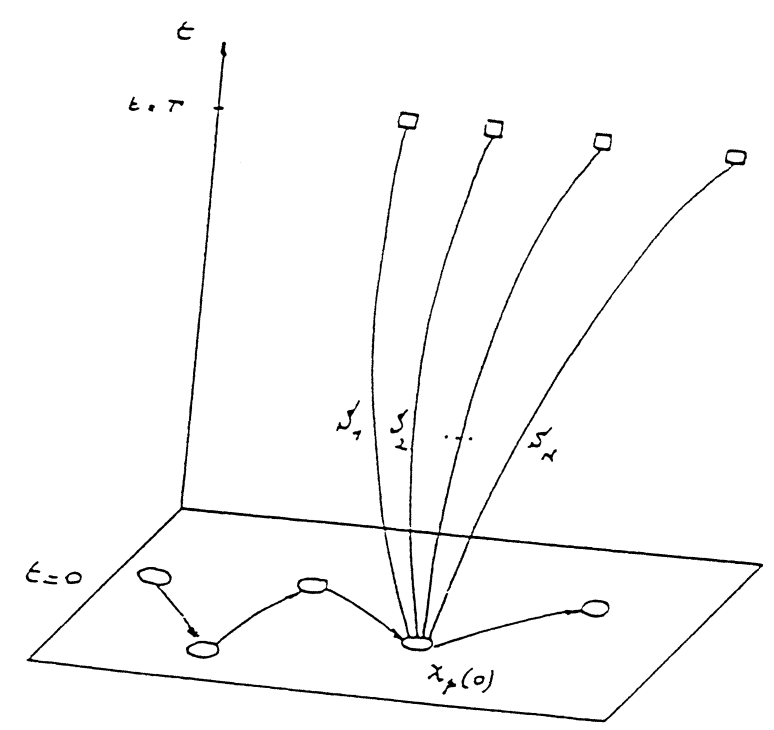

Fig. 1. Calculus of a particular state variable $x_{\mathrm{p}}$ at horizon $T$ according to the different strategies.
European countries. This imposed benchmark has changed many national options and strategies on a purely intuitive basis. A better understanding of the consequences of those intuitive decisions made by politicians in several countries could have averted some undesirable consequences showing up in present days, e.g. with respect to increasing unemployment.

To avoid risky or dangerous strategies, longterm test have to be performed (Kunsch, 1996). These tests discard all paths which move into unacceptable directions, e.g. if some variable would miss by far the preset objectives, or if there is a risk of getting trapped in unfavourable attractor basins, like for instance high levels of public debt, etc.

It is well known that in nonlinear systems multiple stable solutions may co-exist (see Glendinning, 1994). For a given set of parameters, the system may converge to different attractors depending on the initial conditions, or during fluctuations which bring the system close to the interface between two basins (separatrix). The typology and stability character of attractors may also change if the system is submitted to bifurcation. The longterm test should identify the risk of getting trapped into unfavourable or irreversible attractor basins, either by crossing a separatrix or by variable bifurcations generating new attractors with detrimental properties (Kunsch and Chevalier, 1998).

The long-term simulations and tests provide only trends or patterns of behaviour which can prove to be wrong, should some unexpected external event bring major changes. Therefore, the proposed control of structure must be sufficiently flexible on a shorter term perspective, in order to cope with an uncertain future. The rapid dynamic evolution of socio-economic systems and the permanent evolution of their structures requires a short term monitoring and control, as well as the consideration of long-term objectives, at the same time. We shall revert to short-term control in step 10 .

\subsection{Step 8: Ranking and selection}

The selection of strategies for controlling a complex socio-economic system can be handled as a multifactor and a multicriteria problem. 
On the one hand, there are several experts and decision makers; each of them might have different opinions on the respective importance or success of given strategies. For example, in the case of a firm, this could be the managers of production, finance, human resources, marketing, etc. On the other hand, each actor will define his own set of weights with respect to different evaluation criteria. In the same example of the firm, a non exhaustive list of criteria could be: revenues, $\mathrm{R}$ and $\mathrm{D}$ expenses, market share, environmental factors, long-term trends of some specific variables, social acceptability or the ecological compatibility of a strategy.

On a macro-economic level, the set of criteria might include inflation, public debt, unemployment level, social security expenses, investment in public goods, GNP, distribution of wealth, environmental indicators, etc. Each of these criteria or objectives will be given a different weight by different political actors.

More formally, let $f_{1}(),. f_{2}(),. \ldots, f_{K}($.$) be the K$ evaluation criteria the experts wish to take into account. Suppose, in addition, that all the evaluations are real numbers expressed on their particular criterion units or real number scores placed on numerical scales, such as $0-10$ or 0 100 , etc. The criteria must be designed in order to be able to take also the patterns into account and not only the final value at horizon $T$. One can include several other criteria such as the duration of transient behaviour or the time to reach a steady state (Gardiner and Ford, 1980), the maximum point, the number of years from initial equilibrium to maximum, number of years from maximum to second minimum, etc. (Andersen and Rohrbaugh, 1992).

In this case, the following dynamic evaluation table (see Table 1) can be set up.

A set of "good" strategies must then be selected among the set of acceptable ones which have passed the long-term test in step 7. The test discards strategies, without any further analysis, on the basis of the suspicion of being unfavourable in the long term, as shown by simulation. Most acceptable strategies will be at the same time efficient, while the reverse is not necessarily true, as some strategies may take unfavourable values or
Table 1

Multicriteria evaluation table

\begin{tabular}{lllllll}
\hline Strategies & $f_{1}()$. & $f_{2}()$. & $\ldots$ & $f_{j}()$. & $\ldots$ & $f_{K}()$. \\
\hline$S_{1}$ & $f_{1}\left(S_{1}\right)$ & $f_{2}\left(S_{1}\right)$ & $\ldots$ & $f_{j}\left(S_{1}\right)$ & $\ldots$ & $f_{K}\left(S_{1}\right)$ \\
$S_{2}$ & $f_{1}\left(S_{2}\right)$ & $f_{2}\left(S_{2}\right)$ & $\ldots$ & $f_{j}\left(S_{2}\right)$ & $\ldots$ & $f_{K}\left(S_{2}\right)$ \\
$\ldots$ & $\ldots$ & $\ldots$ & $\ldots$ & $\ldots$ & $\ldots$ & $\ldots$ \\
$S_{i}$ & $f_{1}\left(S_{i}\right)$ & $f_{2}\left(S_{i}\right)$ & $\ldots$ & $f_{j}\left(S_{i}\right)$ & $\ldots$ & $f_{K}\left(S_{i}\right)$ \\
$\ldots$ & $\ldots$ & $\ldots$ & $\ldots$ & $\ldots$ & $\ldots$ & $\ldots$ \\
$S_{N}$ & $f_{1}\left(S_{N}\right)$ & $f_{2}\left(S_{N}\right)$ & $\ldots$ & $f_{j}\left(S_{N}\right)$ & $\ldots$ & $f_{K}\left(S_{N}\right)$ \\
\hline
\end{tabular}

trajectories with regard to certain criteria (although the long-term test in the previous step would have eliminated most undesired solutions). Because of the conflict between criteria, the selection is not straightforward. An appropriate Multicriteria Decision Aid (MCDA) approach is required. We propose to use the PROMETHEEGAIA ${ }^{3}$ MCDA methodology (Brans, 1996; Brans et al., 1986; Mareschal and Brans, 1988). The way it can be used with the PROMCALC software is now briefly described.

First, preference functions $P_{k}\left(S_{n}, S_{m}\right)$ are defined for the sake of comparing strategy pairs $S_{n}$, $S_{m}$ for all criteria $f_{k}(),. k=1, \ldots, K$. Each function for a given $k$ is defined as being monotonously increasing, in function of the difference of the scores being obtained by the two strategies on each particular criterion. Different shapes of the preference function with the possibility of multiple preference thresholds can be chosen by means of the PROMCALC software. In this way, a lot of flexibility is provided to represent the preferences of strategy makers.

Suppose now that all pairwise comparisons have been performed, providing all function values.

$$
\begin{aligned}
& 0 \leqslant P_{k}\left(S_{n}, S_{m}\right) \leqslant 1 \quad k=1,2, \ldots K \\
& n, m=1,2, \ldots, N .
\end{aligned}
$$

An aggregated preference index over all the criteria is then calculated.

\footnotetext{
${ }^{3}$ PROMETHEE: Preference Ranking Organisation METHod for Enrichment Evaluations. GAIA: Geometrical Analysis for Interactive Aid.
} 
$\Pi\left(S_{n}, S_{m}\right)=\sum_{j=1}^{K} P_{j}\left(S_{n}, S_{m}\right) w_{j}$,

where $w_{j}$ is a weight, measuring the relative importance allocated to criterion $j$. The weights are supposed to be normalized, so that

$\sum_{j=1}^{K} w_{j}=1$.

The following positive and negative dominance flows express the dominating and the dominated character of a strategy $S_{n}$, respectively, over all the other strategies.

$$
\begin{aligned}
& \Phi^{+}\left(S_{n}\right)=\frac{1}{n-1} \sum_{m=1}^{N} \Pi\left(S_{n}, S_{m}\right), \\
& \Phi^{-}\left(S_{n}\right)=\frac{1}{n-1} \sum_{m=1}^{N} \Pi\left(S_{m}, S_{n}\right) ;
\end{aligned}
$$

while the balance of the dominance flow gives the net flow

$\Phi\left(S_{n}\right)=\Phi^{+}\left(S_{n}\right)-\Phi^{-}\left(S_{n}\right)$.

The latter gives a quality measure of the strategy. The higher this value, the better the strategy. Accordingly, PROMETHEE II ranks the strategies in the order of their decreasing net flows. The associated PROMCALC software allows sensitivity analysis on the weights and graphical investigation of the conflicts between the criteria based on Principal Components Analysis by means of GAIA (Brans and Mareschal, 1994).

The procedure, as described here, is a one step decision procedure in which all the criteria are considered at the same time. A more sophisticated "PROMETHEE Group Decision Support System (GDSS)" has been developed recently (Brans et al., 1996; Mareschal et al., 1997). It involves a twostep approach. The first step consists of an individual analysis, performed by each decision maker, using his own evaluation criteria. The second step encompasses an analysis of the conflicts between the decision makers. This procedure can be applied successfully for selecting, in each iteration, the best possible option.

The best compromised strategy chosen among the set of acceptable strategies from step 7 , is se- lected by using either the classical PROMETHEE II procedure, or by the more sophisticated PROMETHEE GDSS technique. Remember that in both cases the selection takes into account the full time interval $(0, T)$ in which dynamic paths have been simulated. Ranking is always subjective, but it must be consistent. PROMETHEE is a tool to foster consistency and to make subjectiveness transparent, at the same time.

\subsection{Step 9: Realization of the selected strategy}

This step concerns the tactical and operational measures to be taken to have the system perform efficiently and effectively in practice. In a firm this will mean for instance that the operations function appropriately, in congruence with strategic objectives. In a macro-economic case it means that all the political decisions resulting from step 6 to 8 will be properly implemented, and pertinent goals will be achieved.

\subsection{Step 10: Definition of the socio-economic core and short term control}

Having discarded undesirable paths of evolution by using the long-term test, and having ranked desirable strategies by MCDA, will not be sufficient. As the future is inherently uncertain, unexpected external events, or sudden changes in the socio-economic structure can make obsolete the whole future strategy. Progressive or catastrophic changes in the structure could be faced. Therefore, on the one hand strategy revisions are necessary, on the other hand short-term adjustments of tactical nature must supplement the long-term planning.

To provide tactical flexibility, the long-term horizon of the model is divided into shorter time intervals as follows.

$0, \tau, \ldots, i \tau, \ldots, T$,

where $i=1, \ldots n$, and $n \tau=T$.

The length of one time interval $\tau$ will be selected according to the nature of the system, and in function of the accuracy which is needed for an 
adequate control. For macro-economic problems, at the level of national policies, a time interval would typically be between one quarter and one year. For short-term control in firms, 1 month to 1 week intervals will be better adapted.

In the methodology proposed here, each elementary time interval $\tau$ would correspond to one iteration period for reassessing the model after having observed the real system. There are occurences however, in which a warning signal issued by built-in watchdogs would call for an immediate reappraisal of the model. In such cases the current iteration period would be shorther than the basic time interval.

On notice, the decision makers must react without undue delays. The warning signal might mean some irreversible sliding into a non-appropriate attractor basin, or a bifurcation to instability by activation of a previously dormant $(+)$ feedback loop. The sooner the problem is faced, the easier it is to avoid snowballing effects and their partly irreversible impetus to failure. If on the contrary delayed action is taken, it will require much more effort to escape from the unfavourable attractor basin. The whole system may in such cases face irreversible damage or structural instabilities (Kunsch and Chevalier, 1998).

To sum up, a new iteration period will be initiated at the end of each interval $\tau$, or sooner in case some warning would be given by built-in watchdogs. Therefore we may say that in practice the control of structure must take place in real time, as continuous observation is followed by suitable action, if needed.

As any socio-economic system generally includes a large number of system variables, it is beyond the observation capacity to monitor, in real time, each one of them. For this reason it is adequate to define a minimum set of critical or essential variables, we have called the socio-economic core. The latter should be defined in such a way that restricting the control to the variables belonging to the core will be sufficient. In other words, to achieve the purpose of the control in conformity with long-term objectives, it is sufficient to monitor the core variables. As the objectives depend on the preferences expressed by the decision makers, the size and content of the core are not entirely defined by "objective" control necessities, but also partly by subjective judgements. Nevertheless several rules are applicable when defining the core.

1. All variables necessary to represent the objectives considered by the decision makers belong to the socio-economic core.

2. At least one system variable belonging to each one of the critical positive feedback loops must be selected in the core.

3. All watchdog variables and directly linked critical variables (in the meaning described below) belong to the core.

As the core includes a limited number of variables, it will provide a clear, synoptic and sufficient representation of the system. It will help the decision makers and analysts in the real-time control of the system-in-focus.

By concentrating on the core monitoring, the decision makers will be liberated from the load of paying attention to hundreds of more or less important variables. Therefore it would be a rewarding task for further research to investigate the properties of the socio-economic core and to extend the rules for its construction in specific cases.

Among other ideas, the methodology of the cross-impact matrix is thought to be particularly helpful in this instance. Without going into detail, it can be said that this approach assigns the system variables to four groups, called "active", "passive", "critical" and "inert", according to the corresponding dynamic behaviour manifested in the system. The GAMMA software package has been used for providing this classification of variables (Schwaninger, 1997). It can be easily shown that the highly "critical" and "active" variables belong to the core.

At the beginning of a new iteration period the core might have to be modified. Some variables would be withdrawn, while some new ones would be included, depending on the evolution of the structure observed in the system and the possible shifts in objectives as defined by the decision makers.

To fix the idea, let us define the following core variables.

$z_{1}, z_{2}, \ldots, z_{l}, \ldots, z_{L}$ 
Forecasts of their dynamic evolution in a given strategy will be provided by the simulations described in step 7.

\subsection{Step 11: Analysis of the deviations}

At the end of each iteration period, or as soon as a watchdog is giving a warning signal, the decision maker will observe the real time behaviour of the core variables and compare it with plans, assumptions and objectives, or predictions, respectively. Doing so the following Table 2 will be set up.

Technically, the use of advanced mathematical methods of time series analysis, e.g. based on Bayesian algorithms, as proposed under the term "CyberFilter", could prove fertile (for details, see Beer, 1975; Espejo et al., 1996). CyberFilter does not only analyse absolute deviations between values, but dynamic modes of a variable's behaviour, such as stable, transient, unstable, slope down or step up.

The case of monitoring and analysing deviations of the subset of variables and parameters concerning critical assumptions is what an early warning system is all about. Pertinent observation and provision of data must be an on-going task which can be delegated to those instances which can build up an appropriate expertise most effectively. For example, the best grasp on assumptions about exogeneous market forces is often with account managers or market researchers.

The observed deviations in the core variables can be explained in three different ways:
1. Inaccuracy of the mental representation which fails to capture the most important dynamic mechanisms of the real system.

2. Inaccuracy of the model. Socio-economic systems are complex or hypercomplex as said in the introduction. Any model, as sophisticated as it may be, will be a simplification of the realityin-focus. Certain generative factors may therefore be inadequately represented.

3 . The economic structures influencing the system are in perpetual evolution. They may be experiencing significant changes under the impact of other external variables or systems not represented in the model. Some of these changes may have been unpredictable before they did occur, even despite proper monitoring of critical assumptions.

A graphical display of the observed differences will facilitate their interpretation in term of these three shortcomings of the model (see Fig. 2).

This step completes the current iteration period. A decision branching with three alternatives occurs as a consequence of the analysis of differences.

$A$. The deviations observed in the critical variables of the core are considered to be non-significant: The system may be considered as being under control. Also the model is sufficiently accurate for further evaluations, and the same strategy could in principle continue being pursued at the end of the next period (at least if no watchdog gets active before that time). Eventually the economic core can be updated in this case due to the learning process, the decision makers have gone through, while observing the system. They can also reconsider some priorities in their objectives used in their evaluation of the strategies in the PROME-

Table 2

Deviations between planned and observed values/patterns of the core variables

\begin{tabular}{lllllllllll}
\hline & $z_{1}$ & $Z_{1}$ & $\Delta_{1}$ & $z_{2}$ & $Z_{2}$ & $\Delta_{2}$ & $\ldots$ & $z_{L}$ & $Z_{L}$ \\
\hline$t=0$ & $z_{1}\left(t_{0}\right)$ & $Z_{1}\left(t_{0}\right)$ & 0 & $z_{2}\left(t_{0}\right)$ & $Z_{2}\left(t_{0}\right)$ & 0 & $\ldots$ & $z_{L}\left(t_{0}\right)$ & $Z_{L}\left(t_{0}\right)$ & 0 \\
$\tau$ & $z_{1}(\tau)$ & $Z_{1}(\tau)$ & $\Delta_{1}(\tau)$ & $z_{2}\left(t_{1}\right)$ & $Z_{2}(\tau)$ & $\Delta_{2}(\tau)$ & $\ldots$ & $z_{L}(\tau)$ & $Z_{L}(\tau)$ & $\Delta_{\mathrm{L}}(\tau)$ \\
$2 \tau$ & $z_{1}(2 \tau)$ & - & - & $z_{2}(2 \tau)$ & - & - & $\ldots$ & $z_{L}(2 \tau)$ & - & - \\
$\ldots$ & $\ldots$ & $\ldots$ & $\ldots$ & $\ldots$ & $\ldots$ & $\ldots$ & $\ldots$ & $\ldots$ & $\ldots$ & $\ldots$ \\
$\ldots$ & $\ldots$ & $\ldots$ & $\ldots$ & $\ldots$ & $\ldots$ & $\ldots$ & $\ldots$ & $\ldots$ & $\ldots$ & $\ldots$ \\
$T$ & $z_{1}(T)$ & - & - & $z_{2}(T)$ & - & - & $\ldots$ & $z_{L}(T)$ & - & - \\
\hline
\end{tabular}

$z_{l}, l=1,2, \ldots L$ are the predictions, $Z_{1}$ the corresponding observations available at the end of the iteration period $l$, and $\Delta_{l}$ the observed deviations between them. 


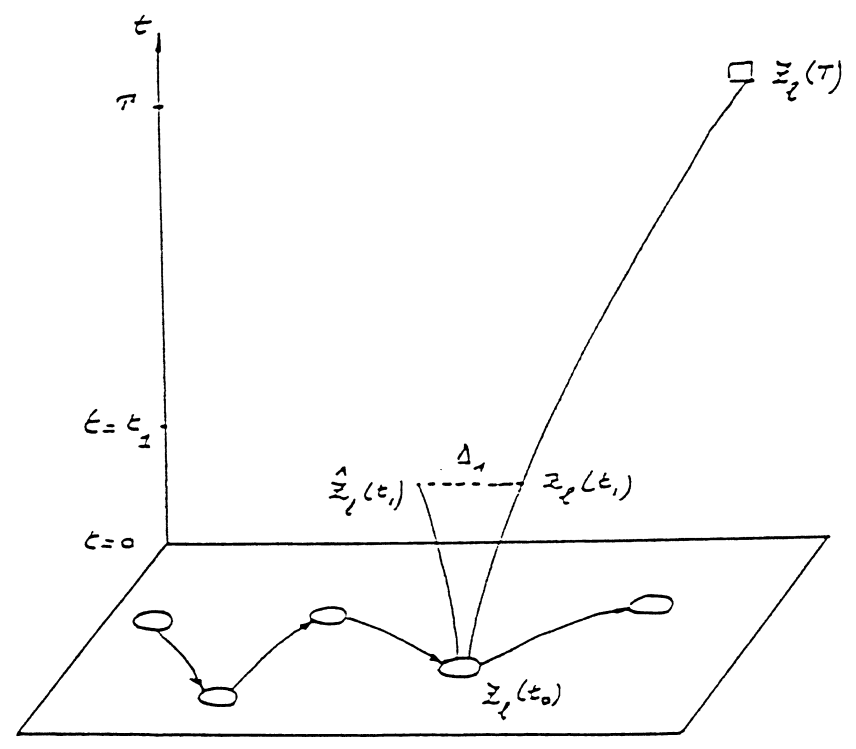

Fig. 2. Deviations between plans/objectives and realisations at time $t_{1}$.

THEE procedure. This would bring us back either to step 10 (definition of the core) or step 8 (selection of an appropriate strategy with the help of PROMETHEE II), before a strategy would be implemented in the next period.

B. Major deviations of some critical variables in the core have been detected: This will be a frequent case in real time control. As a subsidiary branching, one has then to further investigate which one of the three causes for discrepancies has to be taken into consideration, as discussed above.

A.1. The mental representation is not sufficiently accurate: Branching back to step 1 will be necessary, following again the whole decision chain.

A.2. The model is not sufficiently accurate: Branching back to step 5 (modelling and validation) will be necessary, in order to make adjustments in the quantitative model. This may also impinge on the ensuing steps of strategy definitions; e.g. new levers of control or different thrusts may have to be found. New computations will then be necessary to update the input to the long-term test and the MCDA evaluations.

A.3. Some exogeneous occurrence has fundamentally changed the system structure: Again, the decision makers will have to redesign their shared mental representation and the model by tracking back to step 1 for the next iteration period, taking into account the changing environment and the new information.

\section{Conclusion}

The proposed methodology conveys a new path to controlling, under real time conditions, the evolution of hypercomplex socio-economic systems. It is an interactive approach combining the principles of System Dynamics, control theory and the PROMETHEE MCDA tool. The whole procedure is summarised in a "streamlined" (i.e. slightly simplified by abstracting from many possible feedback loops) flow chart (Fig. 3).

Each iteration period consists of a long term planning phase, and a control phase in real time. The learning process is permanent, due to the observation of the system and continual adaptation:

- in the planning phase, the progressive acquisition of experience contributes to enrich the mental representation and the influence diagram by making them more accurate in representing the reality of the observed complex system. Also a specific long-term test discards strategies which 


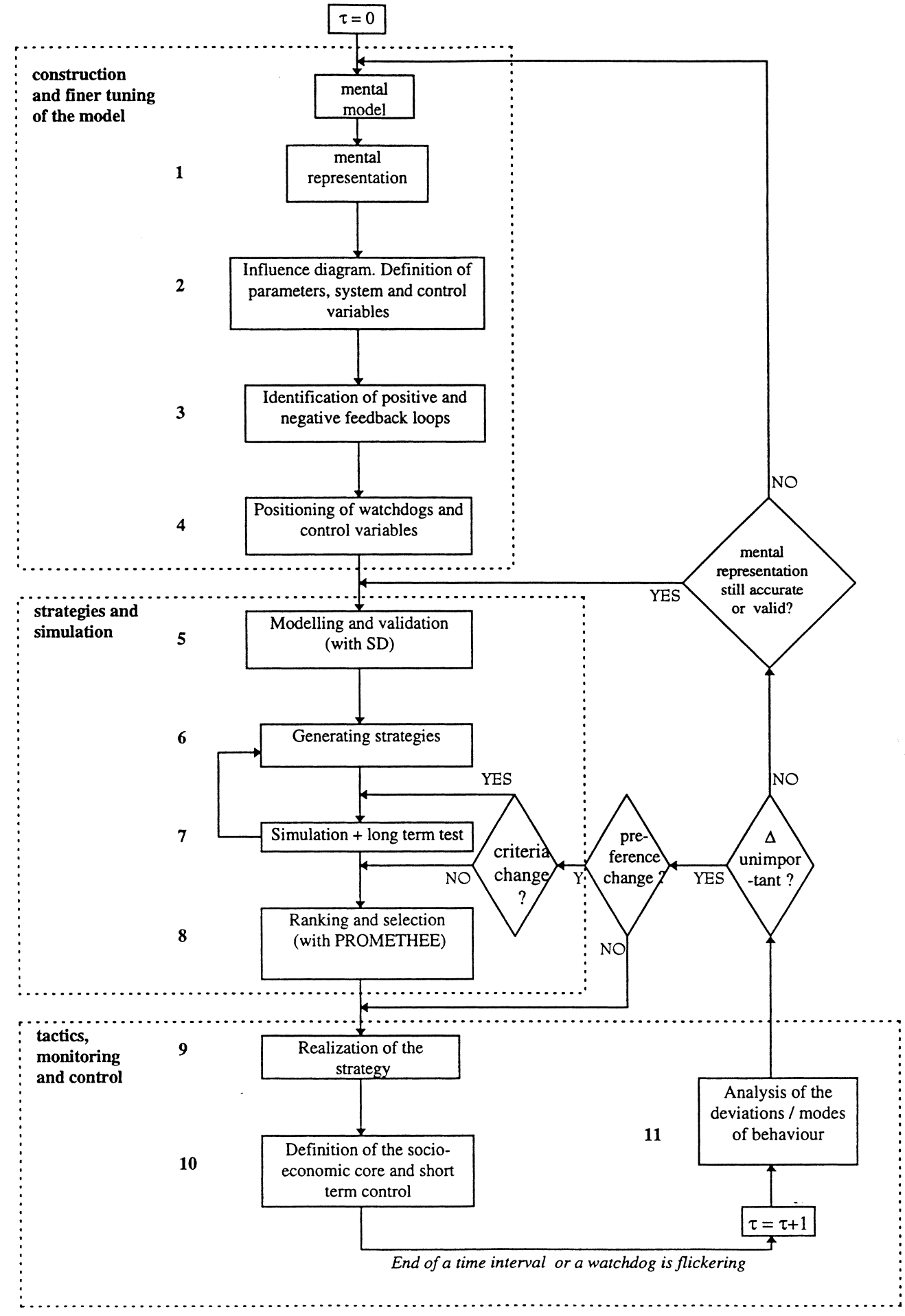

Fig. 3. Flow chart. 
are unacceptable due to the threat of evolutions towards risky attractor basins;

- in the control phase, the observation of changing environment and structures will allow for flexible responses in real time despite inherent limitations of knowledge (Kunsch, 1996; Kunsch and Chevalier, 1998).

The proposed approach seems to be well applicable in practice, as a considerable experience is available in SD and MCDA techniques, including GDSS, respectively. The methodologies combined in the mode proposed here are, in principle, highly complementary. Therefore, this methodology could be a significant contribution to strategymaking at both the societal and the organizational levels. While some additional work on the theoretical foundations of process control is being accomplished by the authors, it is their aim to apply this new concept of control to real world applications in the micro- and macro-economic fields.

Forthcoming papers will document this ongoing research.

\section{References}

Andersen, D.F., Rohrbaugh, J., 1992. Some conceptual and technical problems in integrating models of judgement with simulation models. IEEE Transactions on Systems, Man and Cybernetics 22 (1), 21-34.

Barlas, Y., 1996. Formal aspects of model validity and validation in system dynamics. System Dynamics Review 12 (3), 183-210.

Beer, S., 1975. Platform for Change. Wiley, Chishester, UK.

Brans, J.P., 1996. The space of freedom of the decision-maker, Modelling the human brain. European Journal of Operational Research 92, 593-602.

Brans, J.P., Macharis, C., Marechal, B., Mariame, M., 1996. A two-stage PROMETHEE-GAIA based procedure for group decision support. Working Paper VUB, STOOTW/21, pp. $1-16$.

Brans, J.P., Mareschal, B., Vincke, P.H., 1986. How to select and how to rank projects: The PROMETHEE method. Europeon Journal of Operational Research 24, 228-238.

Brans, J.P., Mareschal, B., 1994. The PROMCALC-GAIA decision support system for multicriteria decision aid. Decision Support Systems 12 (4/5), 297-310.

Brans, J.P., Vincke, P.H., 1985. A preference ranking organisation method: The PROMETHEE method for MCDM. Management Science 31 (6), 647-656.
Checkland, P., 1981. Systems Thinking, Systems Practice. Wiley, Chichester.

Daellenbach, H., 1994. Systems and Decision Making: A Management Science Approach. Wiley, Chichester.

Eden, C., 1994. Cognitive mapping and problem structuring for system dynamics model building. System Dynamics Review $10(2 / 3), 257-276$.

Espejo, R., Schuhmann, W., Schwaninger, M., Bilello, U., 1996. Organizational Transformation and Learning: A Cybernetic Approach to Management. Wiley, Chichester.

Forrester, J.W., 1968. Principles of Systems. Wright-Allen Press, Cambridge, MA.

Forrester, J.W., 1987. Nonlinearity in High-order Models of Social Systems. European Journal of Operational Research 30, 104-109.

Forrester, J.W., 1992. Policies, Decision and Information Sources for Modeling. European Journal of Operational Research 59, 42-63.

Forrester, J.W., Senge, P.M., 1980. Tests for building confidence in system dynamics models. TIMS Studies in the Management Sciences 14, 209-228.

Gardiner, P.C., Ford, A., 1980. Which policy run is best and who says so? TIMS Studies in the Management Sciences 14, 241-257.

Glendinning, P., 1994. Stability, Instability and Chaos. Cambridge University Press, Cambridge.

Kenis, D., 1995. Improving Group decisions: Designing and Testing Techniques for Group Decision Support Systems Applying Delphi Principles. Utrecht Proefschrift, Utrecht.

Kunsch, P.L., 1996. Dynamical aspects of the Decision Support system Process. IFORS Conference, Vancouver (to be submitted).

Kunsch, P.L., Chevalier, A., 1998. A multiple attractor dynamics in socio-economic decision problems. European Journal of Operational Research (this issue).

Lemoigne, J.L., 1995. La modelisation des systèmes complexes. Dunod, Paris.

Macharis, C., Brans, J.P., Mareschal, B., 1997. The GDSS PROMETHEE procedure. Working paper, VUB CSOO no. 277 , accepted for publication in the Journal of Decision Systems.

Mareschal, B., Brans, J.P., 1988. Geometrical representation for MCDM the GAIA procedure. European Journal of Operational Research 34, 69-77.

Meadows, D.H., 1980. The Unavoidable a Priori. In: Randers, J. (Ed.), Elements of the System Dynamics method. Productivity Press, pp. 23-57.

Randers, J., 1980. Guidelines for Model Conceptualization. In: Randers, J. (Ed.), Elements of the System Dynamics Method. Productivity Press, Cambridge, MA, pp. 117-139.

Richardson, G.P., Andersen, D.F., 1995. Teamwork in group model building. System Dynamics Review 11, 113-137.

Schwaninger, M., 1997. Integrative systems methodology, framework and application. Transactions in Operational Research 4 (4), 109-123. 
STELLA, II., 1994. Technical Documentation. Copyright High Performance System, Hanover.

Sterman, J.D., 1994. Learning in and about complex systems. System Dynamics Review 10 (2/3), 291-330.

Vennix, J.A.M., Gubbels, J.W., Post, D., Poppen, H.J., 1990. A Structured approach to knowledge elicitation in conceptual model building. System Dynamics Review 6, 194-208.
Vennix, J.A.M., 1996. Group Model Building. Facilitating Team Learning Using System Dynamics. Wiley, Chichester. Legasto, A.A., Forrester, J.W., Lyneis, J.M. (Eds.), 1980. System Dynamics: TIMS Studies in the Management Sciences, vol.14. North-Holland, New York. 